DEMONSTRATIO MATHEMATICA

Vol. XVII No 1

Andrzej Nowicki

\title{
PRIME IDEAL STRUCTURE IN ADDITIVE CONSERV'ATIVE SYSTEMS
}

In this paper we prove that the set of prime ideals in an additive conservative eyetem is a epectral space and that every morphism of additive conservative systems induces a opeotral map.

Throughout this paper all rings are commutative with identity and all ring homomorphisms preserve the identity.

An additive conservative system ([4], [7]) is a pair $(R, M)$, where $R$ is a ring and $M$ is a set of 1deals of $R$ satisfying the following conditions:

A1. The intersection of anj set of elements of $M$ is an element of $M$.

A2. The union of any nonempty set, totally ordered by inclusion, of elements of $\mathcal{M}$ is an element of $\mathcal{M}$.

A3. The sum of any two 1deals of $M$ is an element of $M$.

Let $(R, M)$ be an additive system. The elements of $M$ are called $M$-ideals. By A1 applied to the empty set of elements of $M, R$ is an $M$-ideal. If $E$ is a subset of $R$, then by [E] we denote the omallest M-ideal containing $\mathrm{B}$. It is clear, that if $A$ and $B$ are ideals in $R$ then $[A+B]=[A]+[B]$.

If $(R, M)$ and $(S, \mathcal{N})$ are additive conservative systems then a ring homomorphisn $f: R \rightarrow S$ will be callod a morphism of additive conservative systems $(R, M)$ and $(S, \mathcal{N})$ iff the inverse images of $\mathcal{N}$-ideals are M-ideals.

L e $m a$ 1. Let $(R, M)$ be an additive conservative system and let $E$ be a subset of $R$. If $x \in[E]$, then there exists a finite subset $T \in E$ such that $x \in[T]$. 
P r 0 o 1 . Seo [7] ar [4].

I $e \mathrm{~m}=$ 2. Iet $(R, M)$ be an additive conservative gystom and lot $A$ be an 1deal of $R$. Then $A$ ig an M-ideal if and only if for every $x \in R$ the oondition $x \in A$ implies [ $x$ ] $A$. P r o o f The necoseity of the condition is obvious. To prove suffioleney wo show that $[A] \subset A$. Let $x \in[A]$, then, by Lemma 1 , we have $x \in\left[a_{1}, \ldots, a_{n}\right]$, for some $a_{1}, \ldots, a_{n} \in A$. since $\left[a_{1}, \ldots, a_{n}\right]=\left[a_{1}\right]+\ldots+\left[a_{n}\right]$ and $\left[a_{1}\right] c A$, for $1=1, \ldots, n$, thus $x \in A$.

For an ring $R, S p e o(R)$ will denote the sot of prime ideala in $R$ with the Zariakl topology ( [1] p.125). If $B$ is a subset of $R$, then by $V(E)$ we denote the set of prime ideals of $R$ containing $B$ and by $D(B)$ wo denote the set $S$ pec $(R) \backslash V(B)$.

Let $(R, M)$ bo an additive coneervative syetem. The set of prime $M-1 d e a l e$ in $R$ will be denoted by $S p e o(R, M)$ and will be oalled the prime spectrum of $(R, M)$. As a topological spooe, 1t has the subspace topology from $S p e c(R)$, so that the closed sets in $\operatorname{Spec}(R, M)$ are of the form $V(E) \cap S p e o(R, M)$, where $B$ is a subset of $R$.

I $\theta \mathrm{m}$ a 3. If $(R, M)$ is an additive conservativo ojetem, then

$$
\operatorname{Speo}(R, M)=\bigcap\{D(r) \cup V([r]), \quad r \in R\} .
$$

$P r \circ 0 f$. Let $P \in S p e c(R, M), r \in R$. If $r \& P$ then $P \in D(r)$. If $r \in P$, then $[r] \subset P$. Therefore, for overy $r \in R$, wo have $S$ pec $(R, M) \subset D(r) \cup V([\mathbf{r}])$. Conversely, assume that $P \in D(r) \cup V([r])$, for anj $r \in R$. We prove that $P \in M$. Let $r \in P$. Then $P \notin D(r)$ and thus $P \in V([r])$. Therefore $[r] \subset P$ and, by Lemme 2, we have $P \in M$.

Let $f:(R, M) \rightarrow(S, N)$ be a morphism of additive conservative eystems. Then $f$ induoes a continuous mapping $a_{f}: S p e o(S) \rightarrow S p e o(R)$ via $a_{f}(P)=f^{-1}(P)$, where $P \in S p e o(S)$. Since $a_{f}(\operatorname{Spec}(S, N)) \subset \operatorname{Spoc}(R, M)$, we have a continuous mapping

$$
b_{f}: S p e o(S, N) \longrightarrow S p e o(R, M) \text {, }
$$

given by $b_{f}(P)=a_{f}(P)$, for ang $P \in S p e c(S, \mathcal{N})$.

$$
-10 \text { - }
$$


Reoall from ([5]) that a topologieal opace $X$ is called spootral if it is $T_{0}$ and guesi-compact, the quesi-compact open subsets ere olosed under finite interseotion and form an open basis, and overy nonempty irroducible olosed subset has a seneric point. Moroover, a continuous map of epectral opaoes is called speotral if the inverse image of quasi-compact open sets are quas1-oompact. It is woll known, that for every ring $R$, Speo(R) 18 a spectral spaos, and, for overy ring homomorphism $f$ a 18 a seotral map.

I 0 a 4. Iat $I$ be a subspace of a epootral space $X$. Bvery guasi-compact open set in $I$ is of the form $U \cap Y$, whore $U$ is a quasi-oompaot open $80 t$ in $X$.

$P$ I 0 o $I$. Iot $V$ be a opon sot in $X$ anch that $\nabla \cap Y$ Is a quasi-oonpaot set in $J$, and lot $V=U_{U_{1}}$, where $U_{1}$ are

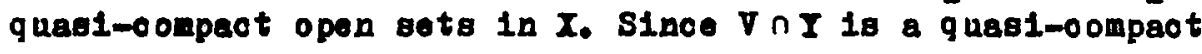
set, we here $V \cap I=\left(U_{1} u \ldots U_{1_{n}}\right) \cap I$. The set $U_{1_{1}} U \ldots U U_{1_{n}}$ 18 guasi-compact open in $x$, beceuse, for overy topologloal apace, the quasi-oompact open subsets are closed under finite an1on:

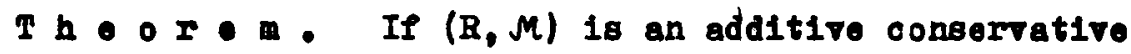
eyatem then Speo $(R, M)$ is a epeotral opace. If $f:(R, M) \longrightarrow$ $\longrightarrow(S, M)$ is a morphtsm of additive oonservative ejotems then be is a speotral map.

P r 0 o $I$. Iomas 3 implies that $S p o o(R, M)$ is a olosed sot in the patch topology on Spoo(R) (aee [5] p.45). It follows that Speo(R, $M$ ) 18 a apeotral aubobjoot of Spoo(R) (seo [5] $\left.p_{0} 45\right)$, and therefore $S p e 0(R, M)$ is a speotral spaoe.

Iot $I,(R, M) \rightarrow(S, N)$ be a morphism of additire conaerrative ejetems and let $G$ bo a quasi-oompaot open set in Speo $(S, M)$. By Lomms 4 , there exists a quasi-compact open sot $U$ in Spoo(S), euch that $G=U \cap S p e o(S, N)$. Since $b_{f}^{-1}(G)=$ $=\left(a_{f}^{-1}\right)(U) \cap \operatorname{Spec}(R, M)$ and $a_{f}^{-1}(U)$ is a quasi-compact open set in $S$ pec $(R)$, and aince $S$ pec $(R, M)$ is a epectral subobject of $S$ peo $(R)$, then $b_{f}^{-1}(G)$ is a quasi-compact open eot in $S p e c(R, M)$. 
Cor 011 a $\mathrm{I}$. Let $f:(R, M) \rightarrow(S, N)$ be a morphism of additive conservative systems. Then there exist rings $R^{\prime}$ and $S^{\prime}$ and a ring homomorphism $f^{\prime}: R^{\prime} \rightarrow S^{\prime}$ such that Spec $(R, M)$ is homeomorphic to $\operatorname{Spec}\left(R^{\prime}\right), S p e c(S, N)$ is homeomorphic to $\operatorname{Spec}\left(S^{\prime}\right)$, and $b_{f}$ is equivalent to $a_{f}^{\prime} 1_{0} \theta_{\cdot} b_{f}=$ $=g a_{f^{\prime}} p$, where $p: \operatorname{Speo}(S, \mathcal{N}) \longrightarrow \operatorname{Spec}\left(S^{\prime}\right), q: \operatorname{Spec}\left(R^{\prime}\right) \longrightarrow$ $\longrightarrow$ Speo( $R, M)$ are homeomorphisms.

$P$ I $0 \circ f$. Th1s follows from Theorem and from [5] Th.6. p.51.

$\mathrm{E} \times \mathrm{ampI}$ e $\mathrm{g}:$ 1. Let $\mathrm{B}$ be a ring, $\mathrm{F}$ a set of endomorphisms of the additive group of $R$, and let $M$ be the set of Ideals $A$ in $R$ such that $f(A) \subset A$ for $f \in$ P. Then $(R, M)$ is an additive conservative eyetem.

2. Let $D$ be a set of derivations of $R$ and let $M$ be the set of differential idoels of $R([7])$. Then $(R, M)$, is an additive conservative system. Every differentlal homomorphism is a morphism of additive conservative sjetems. The theorem, for special differential rings, was proved by W.F.Kelgher in [6], and, for arbitrary differsntial ringa, was proved by G.Carra in [3].

3. Let $D$ be a set of higher derivations of $R$ and let $M$ be a set of $D$-ideals ([2], [4]). Then (R, $M$ ) is an additive conservative syatem and every D-homomorphism is a morphism of additive conservative systems.

4. Let $R$ be a graded ring and let $M$ be the set of homogenoous ideals of $R$. Then $(R, M)$ is an additive conservative gystem and every homomorphism of graded rings is a morphism of additive conservative systems.

$R$ e m a r s 1. Lemma 1 is also true without the assumption that $M$ satisfies $A 3$ (see [7], [4]).

2. If $M$ is a set of ideals of $R$ satisfying only $A 1$ and A2 then Lemma 2 is not necessarily true $\theta . g$. Let $R=Z_{2}[X, Y] / A$, where $A$ is an ideal in $Z_{2}[X, Y]$ generated by $X^{2}, X Y, Y^{2}$. Let $x=X+A, y=Y+A$ and $M=\{0,(x),(y),(x+y), R\}$. Then the ideal $(x, y)$ satisfies the condition of Lemma 2 , and it is not $M$-ideal. 


\section{REFERENCES}

[1] N. B o u r b a 1 : Algebre Commutative, (Elem. Math. 27), Paris 1961.

[2] W.C. B T O D, W. K a $\mathrm{B}$, Ideals and higher derivations in commutative rings, Canad. J. Math. 24 (1972) 400-415.

[3] G. C a r a : Sullo spetro differenalele di un anello differenziale, Matematioohe 33 (1978) 1-17.

[4] R.M. C o h n: Syetems 1f idealo, Caned. J. Math. 21 (1969) 783-807.

[5] H. H O o b o r : Primo ideal structure in commutat1ve rings, Trans, Awer. Math. Soc. 141 (1969) 43-60.

[6] H.F. $K \in 18 \mathrm{~h} \theta \mathrm{r}:$ Prime differential 1dealo in difforential rings, Contributious to Algobra: A Collection of Papers Dedicated to Bl11s Kolohin, New Iork 1977.

[7] E. $K \circ I \circ h \perp n:$ Differential algebra and algebraic groupe. New York 1973.

INSTITUTE OF MATHEMATICS, $\pi$. COPERNICUS UNIVERSITY,

87-100 TORUN, POLAND.

Received July 14, 1981. 
